\title{
Improved precision on the experimental $E 0$ decay branching ratio of the Hoyle state
}

T. K. Eriksen, ${ }^{1, *}$ T. Kibédi $\odot,{ }^{1, \dagger}$ M. W. Reed, ${ }^{1}$ A. E. Stuchbery, ${ }^{1}$ K. J. Cook,${ }^{1,2}$ A. Akber, ${ }^{1}$ B. Alshahrani, ${ }^{1,}$ A. A. Avaa,,${ }^{3,4}$ K. Banerjee, ${ }^{1,5}$ A. C. Berriman, ${ }^{1}$ L. T. Bezzina, ${ }^{1}$ L. Bignell, ${ }^{1}$ J. Buete, ${ }^{1}$ I. P. Carter, ${ }^{1}$ B. J. Coombes,,${ }^{1}$ J. T. H. Dowie, ${ }^{1}$ M. Dasgupta, ${ }^{1}$ L. J. Evitts, ${ }^{6,7,8}$ A. B. Garnsworthy, ${ }^{6}$ M. S. M. Gerathy, ${ }^{1}$ T. J. Gray, ${ }^{1}$ D. J. Hinde, ${ }^{1}$ T. H. Hoang, ${ }^{8}$ S. S. Hota, ${ }^{1}$ E. Ideguchi ${ }^{8}$ P. Jones, ${ }^{3}$ G. J. Lane, ${ }^{1}$ B. P. McCormick, ${ }^{1}$ A. J. Mitchell, ${ }^{1}$ N. Palalani,,${ }^{1, \mathbb{I}}$ T. Palazzo, ${ }^{1}$ M. Ripper, ${ }^{1}$ E. C. Simpson, ${ }^{1}$ J. Smallcombe,,${ }^{6, * *}$ B. M. A. Swinton-Bland, ${ }^{1}$ T. Tanaka, ${ }^{1}$ T. G. Tornyi,,${ }^{1+\dagger}$ and M. O. de Vries ${ }^{1}$ ${ }^{1}$ Department of Nuclear Physics, Research School of Physics, The Australian National University, Canberra ACT 2601, Australia

${ }^{2}$ Facility for Rare Isotope Beams, Michigan State University, 640 South Shaw Lane, East Lansing, Michigan 48824, USA

${ }^{3}$ iThemba LABS, National Research Foundation, P.O. Box 722, 7129 Somerset West, South Africa

${ }^{4}$ School of Physics, University of Witwatersrand, Johannesburg, 2000, South Africa

${ }^{5}$ Variable Energy Cyclotron Centre, 1/AF, Bidhan Nagar, Kolkata 700064, India

${ }^{6}$ TRIUMF, 4004 Wesbrook Mall, Vancouver, British Columbia V6T 2A3, Canada

${ }^{7}$ Department of Physics, University of Surrey, Guildford GU2 7XH, United Kingdom

${ }^{8}$ Research Center for Nuclear Physics, Osaka University, Ibaraki, Osaka 567-0047, Japan

(Received 28 May 2020; accepted 29 July 2020; published 17 August 2020)

\begin{abstract}
Background: Stellar carbon synthesis occurs exclusively via the $3 \alpha$ process, in which three $\alpha$ particles fuse to form ${ }^{12} \mathrm{C}$ in the excited Hoyle state, followed by electromagnetic decay to the ground state. The Hoyle state is above the $\alpha$ threshold, and the rate of stellar carbon production depends on the radiative width of this state. The radiative width cannot be measured directly, and must instead be deduced by combining three separately measured quantities. One of these quantities is the $E 0$ decay branching ratio of the Hoyle state, and the current $10 \%$ uncertainty on the radiative width stems mainly from the uncertainty on this ratio. The rate of the $3 \alpha$ process is an important input parameter in astrophysical calculations on stellar evolution, and a high precision is imperative to constrain the possible outcomes of astrophysical models.
\end{abstract}

Purpose: To deduce a new, more precise value for the $E 0$ decay branching ratio of the Hoyle state.

Method: The $E 0$ branching ratio was deduced from a series of pair conversion measurements of the $E 0$ and $E 2$ transitions depopulating the $0_{2}^{+}$Hoyle state and $2_{1}^{+}$state in ${ }^{12} \mathrm{C}$, respectively. The excited states were populated by the ${ }^{12} \mathrm{C}\left(p, p^{\prime}\right)$ reaction at $10.5 \mathrm{MeV}$ beam energy, and the pairs were detected with the electron-positron pair spectrometer, Super-e, at the Australian National University. The deduced branching ratio required knowledge of the proton population of the two states, as well as the alignment of the $2_{1}^{+}$state in the reaction. For this purpose, proton scattering and $\gamma$-ray angular distribution experiments were also performed.

Results: An $E 0$ branching ratio of $\Gamma_{\pi}^{E 0} / \Gamma=8.2(5) \times 10^{-6}$ was deduced in the current work, and an adopted value of $\Gamma_{\pi}^{E 0} / \Gamma=7.6(4) \times 10^{-6}$ is recommended based on a weighted average of previous literature values and the new result.

Conclusions: The new recommended value for the $E 0$ branching ratio is about $14 \%$ larger than the previous adopted value of $\Gamma_{\pi}^{E 0} / \Gamma=6.7(6) \times 10^{-6}$, while the uncertainty has been reduced from $9 \%$ to $5 \%$. The new result reduces the radiative width, and hence $3 \alpha$ reaction rate, by $11 \%$ relative to the adopted value, and the uncertainty to $6.1 \%$. This reduction in width and increased precision is likely to constrain possible outcomes of astrophysical calculations.

DOI: 10.1103/PhysRevC.102.024320

\section{INTRODUCTION}

The synthesis of heavier elements in the universe is initiated by the $p p$-chain reactions in hydrogen burning stars, where four protons are ultimately converted into one $\alpha$ particle

*Present address: Department of Physics, University of Oslo, $\mathrm{N}-0316$ Oslo, Norway.

†Corresponding author: Tibor.Kibedi@anu.edu.au

${ }^{\star}$ Present address: King Khalid University, Department of Physics, Faculty of Science, Abha 61413, Saudi Arabia.

§Present address: Nuclear Futures Institute, Bangor University, Bangor, Gwynedd, LL57 2DG, United Kingdom.

"Present address: University of Botswana, 4775 Notwane Rd., Gaborone, Botswana. with the release of energy. However, proton capture reactions

\footnotetext{
** Present address: Oliver Lodge Laboratory, University of Liverpool, Liverpool L69 9ZE, United Kingdom.

${ }^{\dagger}$ Present address: Institute for Nuclear Research, The Hungarian Academy of Sciences, Debrecen 4026, Hungary.
} 


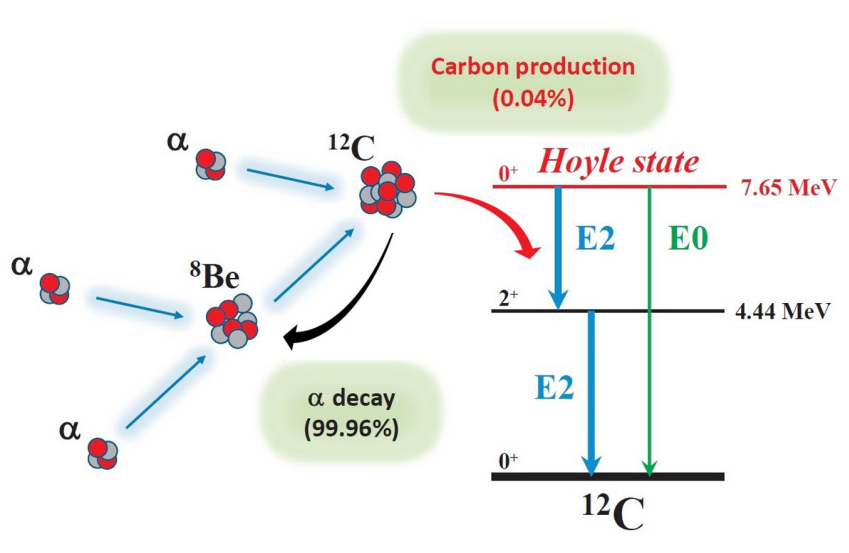

FIG. 1. The $3 \alpha$ process and the decay modes of the Hoyle state.

forming heavier elements are inhibited by the rapid disintegration of ${ }^{8} \mathrm{Be}, T_{1 / 2}=8.2 \times 10^{-17} \mathrm{~s}$ [1], into two $\alpha$ particles, so that no heavier elements are formed in stars at the hydrogen burning stage. It was not known how nucleosynthesis could proceed beyond ${ }^{8} \mathrm{Be}$ until Salpeter suggested that an equilibrium concentration of ${ }^{8} \mathrm{Be}$ can be sustained in a star of sufficient helium concentration and stellar temperature, resulting in a small probability for a third $\alpha$ particle to fuse with the ${ }^{8} \mathrm{Be}$ and form ${ }^{12} \mathrm{C}$ [2]. Carbon production was thus suggested to occur via a sequential fusion of three $\alpha$ particles, $\left(\alpha+\alpha \rightarrow{ }^{8} \mathrm{Be}\right)+\alpha \rightarrow{ }^{12} \mathrm{C}^{*}$, now commonly known as the $3 \alpha$ process. The stellar conditions required for the $3 \alpha$ process are fulfilled at the end of the hydrogen burning stage, due to gravitational contraction of the helium produced by the $p p$-chain reactions. The $0_{2}^{+}$state at $7.65 \mathrm{MeV}$ above the ground state in ${ }^{12} \mathrm{C}$ is crucial for the $3 \alpha$ process, as it acts as a resonance for $s$-wave $\alpha$ capture at the relevant stellar temperatures. Without this resonant state, the cross section for the sequential $3 \alpha$ process would be too small to produce the observed carbon abundance in the universe. The resonant state was predicted by Fred Hoyle [3] before the first experimental observations [4,5], and became known as the Hoyle state.

The Hoyle state energy exceeds the $\alpha$ decay threshold, and it disintegrates back to ${ }^{8} \mathrm{Be}+\alpha$ or $3 \alpha \sim 99.96 \%$ of the time [6]. Stable carbon is only formed in $\sim 0.04 \%$ of the $3 \alpha$ reaction instances, by electromagnetic decay to the ground state. Figure 1 provides a schematic illustration of the formation and various decay modes of the Hoyle state. Direct disintegration to three $\alpha$ particles occurs very rarely, as is indicated by recent measurements, which provide upper limits of $0.043 \%[7,8]$ and $0.019 \%$ [9] for this decay mode relative to the total $\alpha$ break-up. The branching ratio of direct vs. sequential decay is important for structure studies of the Hoyle state, but it is not relevant in the context of stellar carbon formation because the contribution from direct fusion of three $\alpha$ particles is negligible.

The carbon production rate can be described by the resonance equation [10]

$$
r_{3 \alpha}=4 \sqrt{27} \times \frac{N_{\alpha}^{3} \pi^{3} \hbar^{5}}{M_{\alpha}^{3} k_{\mathrm{B}}^{3} T^{3}} \times \frac{\Gamma_{\alpha} \Gamma_{\mathrm{rad}}}{\Gamma} \times e^{-\left(E_{3 \alpha} / k_{\mathrm{B}} T\right)},
$$

where $N_{\alpha}$ and $M_{\alpha}$ are the number density and mass of the interacting $\alpha$ particles, and $\Gamma, \Gamma_{\alpha}$, and $\Gamma_{\text {rad }}$ are the total, $\alpha$ decay, and radiative decay widths of the Hoyle state, respectively. Furthermore, $E_{3 \alpha}=0.38 \mathrm{MeV}$ is the energy released in the break-up of the Hoyle state, and $\hbar, k_{\mathrm{B}}$, and $T$ are the reduced Planck constant, the Boltzmann constant, and the temperature, respectively. Since the Hoyle state decays mainly by $\alpha$ emission, $\Gamma \approx \Gamma_{\alpha}$, and Eq. (1) may be simplified into the expression

$$
r_{3 \alpha} \propto \frac{\Gamma_{\mathrm{rad}}}{T^{3}} \times e^{-\left(E_{3 \alpha} / k_{\mathrm{B}} T\right)},
$$

which shows that the carbon production rate depends directly on the radiative width of the Hoyle state. Due to the sequential nature of the $3 \alpha$ process and the short half-life of ${ }^{8} \mathrm{Be}, \Gamma_{\text {rad }}$ cannot be measured directly. However, it can be deduced indirectly by three independently measured quantities [shown in square brackets in Eq. (3)] according to

$$
\Gamma_{\mathrm{rad}}=\left[\frac{\Gamma_{\mathrm{rad}}}{\Gamma}\right] \times\left[\frac{\Gamma}{\Gamma_{\pi}^{E 0}}\right] \times\left[\Gamma_{\pi}^{E 0}\right],
$$

where $\Gamma_{\pi}^{E 0}$ is the partial $E 0$ pair decay width. The current recommended radiative width obtained from Eq. (3) is $\Gamma_{\text {rad }}=$ $3.7(4) \mathrm{meV}$ [6], which has an uncertainty of $10 \%$. The uncertainties on the individual quantities are $2.5 \%, 9.0 \%$, and $3.2 \%$ for $\Gamma_{\mathrm{rad}} / \Gamma[11-18], \Gamma / \Gamma_{\pi}^{E 0}[19-23]$, and $\Gamma_{\pi}^{E 0}[24]$, respectively, hence the uncertainty on the radiative width stems mainly from the challenges of measuring $\Gamma_{\pi}^{E 0} / \Gamma$. The goal of the present work was to extract $\Gamma_{\pi}^{E 0} / \Gamma$ by a new measurement with improved precision.

\section{METHOD}

The E0 pair branching ratio of the Hoyle state was determined from electron-positron pair measurements of the ground state transitions of the first and second excited states in ${ }^{12} \mathrm{C}$, shown in Fig. 1, based on the procedure reported by Alburger [23]. In a ${ }^{12} \mathrm{C}\left(p, p^{\prime}\right)$ experiment, the number of experimentally measured $E 0$ pairs following decay of the Hoyle state can be expressed as

$$
N_{\pi}^{E 0}=N_{p}\left(0_{2}^{+}\right) \times \frac{\Gamma_{\pi}^{E 0}}{\Gamma} \times \epsilon_{\pi}^{E 0},
$$

where $N_{p}\left(0_{2}^{+}\right)$is the number of protons populating the Hoyle state, $\Gamma_{\pi}^{E 0} / \Gamma$ is the $E 0$ pair decay branching ratio, and $\epsilon_{\pi}^{E 0}$ is the pair detection efficiency of the $7.65 \mathrm{MeV} E 0$ transition. Similarly, for the $E 2$ transition de-exciting the $4.44 \mathrm{MeV} 2_{1}^{+}$ state in ${ }^{12} \mathrm{C}$, the expected number of pairs is

$$
\begin{aligned}
N_{\pi}^{E 2}= & {\left[N_{p}\left(2_{1}^{+}\right)+\left(N_{p}\left(0_{2}^{+}\right) \times \frac{\Gamma_{\text {rad }}\left(0_{2}^{+} \rightarrow 2_{1}^{+}\right)}{\Gamma}\right)\right] } \\
& \times \frac{\alpha_{\pi}}{\left(1+\alpha_{\pi}\right)} \times \epsilon_{\pi}^{E 2} \\
\simeq & N_{p}\left(2_{1}^{+}\right) \times \frac{\alpha_{\pi}}{\left(1+\alpha_{\pi}\right)} \times \epsilon_{\pi}^{E 2},
\end{aligned}
$$

where the second term in the bracket may be omitted because $\Gamma \gg \Gamma_{\text {rad }}\left(0_{2}^{+} \rightarrow 2_{1}^{+}\right)$. The pair decay probability of the $4.44 \mathrm{MeV} E 2$ transition is accounted for by using the theoretical pair conversion coefficient, $\alpha_{\pi}=I_{\pi} / I_{\gamma}$. The $E 0$ pair branching ratio of the Hoyle state may then be expressed 
by rearranging the ratio of Eqs. (4) and (5) as

$$
\frac{\Gamma_{\pi}^{E 0}}{\Gamma}=\frac{N_{\pi}^{E 0}}{N_{\pi}^{E 2}} \times \frac{N_{p}\left(2_{1}^{+}\right)}{N_{p}\left(0_{2}^{+}\right)} \times \frac{\epsilon_{\pi}^{E 2}}{\epsilon_{\pi}^{E 0}} \times \frac{\alpha_{\pi}}{\left(1+\alpha_{\pi}\right)} .
$$

Hence, to deduce the $E 0$ pair decay branching ratio one needs to measure the pair transitions and proton population of the two excited states in question. Furthermore, the angular distribution of the $E 2 \gamma$ decay must be known to account for alignment of the $2_{1}^{+}$state, which can affect the observed pair decay intensity. Measurements of the pair transitions and the proton population ratio of the two excited states, as well as of the angular distribution of the $4.44 \mathrm{MeV} \gamma$ ray de-exciting the $2_{1}^{+}$state, were performed in the present work. The detector efficiency for pair measurements was determined from Monte Carlo simulations, as described in Sec. III B.

\section{EXPERIMENTAL DETAILS}

\section{A. Spectrometer setup}

The experimental setup is located in the Heavy Ion Accelerator Facility (HIAF) at The Australian National University (ANU). Proton beams were delivered by the 14 UD pelletron tandem accelerator [25]. A new spectrometer setup was developed and optimized for pair measurements, based on the existing ANU 2.1 T superconducting solenoid [26]. The main upgrades involved a new baffle system and detector array, which will be described later in this section. The solenoid itself consists of liquid helium cooled NbTi coils, which provide a highly homogeneous and axially symmetric magnetic field, with a uniformity of $-3.7 \% \leqslant \Delta B / B \leqslant$ $+1.6 \%$ within the spectrometer volume [26]. The coil current is computer controlled, and the magnetic field is monitored with a Hall probe. The most effective electron-positron pair measurements are achieved when the solenoid is set up to sample discrete magnetic fields providing maximum transmission of both pair constituents. The optimum magnetic fields depend on the transitions of interest and spectrometer transmission properties, as will be described in Sec. III B. The in-beam sampling is determined by integrated current in the beam dump. Figure 2 provides a cross-sectional illustration

\section{SUPERE PAR SPECTROMEIER}

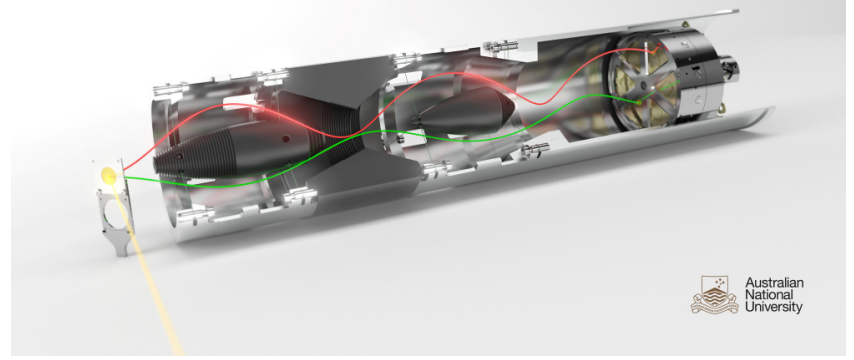

FIG. 2. An illustration of the Super-e pair spectrometer, showing the target, baffle system, and detector array (from left to right). The setup is mounted perpendicular to the beam, which is represented in yellow. An electron-positron pair transmission is indicated by the red and green trajectories. Image courtesy of Thomas Tunningley, ANU. of the Super-e pair spectrometer, revealing its components. The spectrometer is mounted perpendicular to the beam axis, and its dimensions are defined by the solenoid bore diameter and the target-detector distance, which are $\oslash=84.2 \mathrm{~mm}$ and $l=350 \mathrm{~mm}$, respectively.

Starting from the left-hand side of Fig. 2, it can be seen that the target is positioned at $45^{\circ}$ relative to the beam to allow electrons and positrons to be emitted through the rear of the target and into the spectrometer. Electrons and positrons emitted within the acceptance angles and momentum window of the spectrometer are transported through the baffle system and reach the detector plane after following helical trajectories due to the Lorentz force. The axially symmetric baffle system is designed to shield the detector array against $\gamma$ rays emitted from the target, and consists of two axial baffles and a diaphragm made of Heavymet (W-Ni-Fe alloy) coated with a $1 \mathrm{~mm}$ layer of TorrSeal (low vapor pressure epoxy), a low- $Z$ material intended to reduce both the amount of scattering and secondary electron production.

The $\mathrm{Si}(\mathrm{Li})$ detector array, named Miel, consists of six identical, $9 \mathrm{~mm}$ thick sector-shaped $\mathrm{Si}(\mathrm{Li})$ segments, each with an active area of $236 \mathrm{~mm}^{2}$ [27]. When assembled, the segments form an annular array, but are separated by 3-mm-thick, nonmagnetic Heavymet spacers to suppress cross-scattering of electrons and positrons between segments. Cross-scattering of $511 \mathrm{keV}$ annihilation quanta is also suppressed. The assembled detector array can be seen to the right in Fig. 2. The Miel $\mathrm{Si}(\mathrm{Li})$ array may be operated as a single detector by summing the individual spectra of the segments, or in coincidence mode by requiring two or more segments to have fired, which is the case for the pair measurements in the present work. The six segments of Miel provide 15 unique two-segment coincidence combinations. The thickness of the segments allows for full absorption of electrons and positrons up to a kinetic energy of $3.5 \mathrm{MeV}$, which corresponds to a transition energy of 8 $\mathrm{MeV}$ for internal pair formation. Thus, the array is capable of detecting the $7.65 \mathrm{MeV} E 0$ transition from the Hoyle state.

The spectrometer setup is complemented by a HPGe detector used for monitoring the $\gamma$ emission from the target. The detector is positioned at $135^{\circ}$ relative to the beam axis, $1.5 \mathrm{~m}$ away from the target, and has a crystal size of $81 \mathrm{~mm} \times 54 \mathrm{~mm}$ (length $\times$ diameter). Data measured at different magnetic fields may then be normalized to relative sampling and reaction rates by using the peak area of a strong $\gamma$-ray transition in spectra projected with gates on the respective magnetic fields. The same $\gamma$ line is used for all normalizations in a particular experiment. In this work, the strong 4.44 MeV $2_{1}^{+} \rightarrow 0_{1}^{+} \gamma$-ray transition was used for normalization.

The quantities recorded in the current work were the energies and times from the six $\mathrm{Si}(\mathrm{Li})$ segments of Miel, the energy from the HPGe monitor detector, the solenoid control voltage and the Hall probe reading. There were two trigger requirements for storing the information, namely either two $\mathrm{Si}(\mathrm{Li})$ signals in coincidence or a signal from the HPGe monitor detector. The data were stored event by event, and sorted offline. Summed Miel energies, Miel time differences, and the magnetic rigidities of the particles were deduced from the stored quantities. The summed electron-positron pair energy 


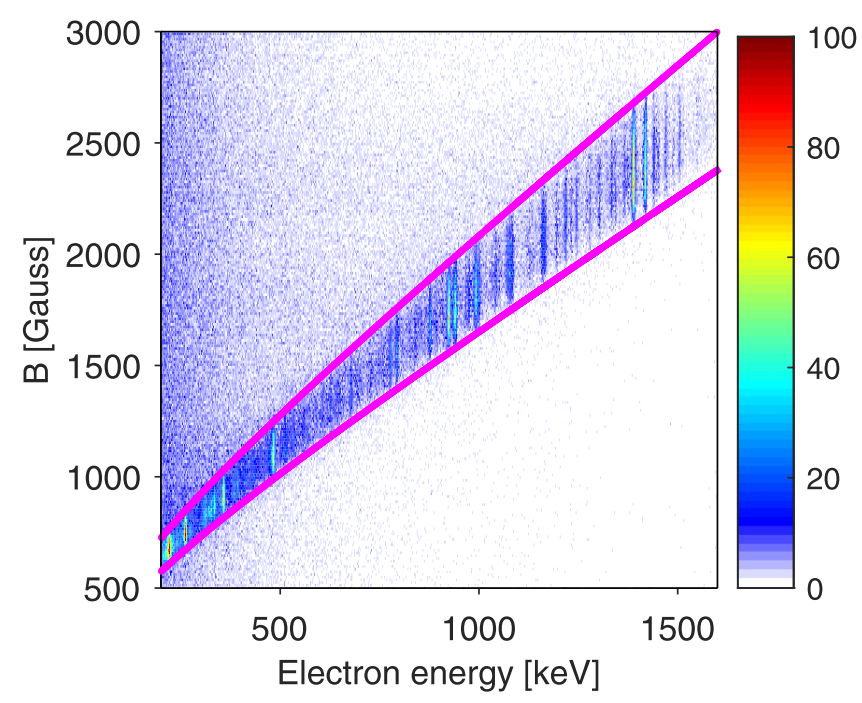

FIG. 3. Energy vs. magnetic field from a ${ }^{170} \mathrm{Lu}$ source conversion-electron measurement, demonstrating the increasing width of the momentum window as a function of magnetic field strength and measured energy. The solid lines indicate the bounds of the momentum window, calculated as described in Ref. [26]. The color scale indicates the number of counts.

could then be projected with gates on the physical momentum window of the spectrometer and prompt time differences, with background subtraction performed by gating on the random time differences.

\section{B. Spectrometer efficiency}

The overall pair detection efficiency depends on the spectrometer transmission and intrinsic detector efficiency. The transmission is determined by the spectrometer acceptance angles with respect to the symmetry axis, $\theta \in\left[15.9^{\circ}, 46.9^{\circ}\right]$, the geometry of the baffle system, and the magnetic field strength. In addition to the directional limits of the acceptance angles, these properties define the physical limits in terms of momentum (the momentum window) for transportation of an electron or positron from the target through the baffle to the detector surface. Particles emitted within the acceptance angles and momentum window are able to reach the detector, while particles outside either will not be transmitted. The width and centroid of the momentum window increases with magnetic field strength, which means that the transmission efficiency of the spectrometer increases with particle energy, and that there is an optimum magnetic field for transportation of a certain particle energy. A magnetic field vs. energy matrix from a singles conversion electron measurement is displayed in Fig. 3, depicting the increasing momentum window as a function of magnetic field and measured energy. The solid lines indicate the limits of the momentum window. An example demonstrating the momentum window for pair measurements is provided in the energy vs. energy matrix shown in Fig. 4. The transmission of an electron-positron pair involves the directional kinematics of two correlated particles, for which the emission is dictated by the energy-angle correlation between the electron and positron.

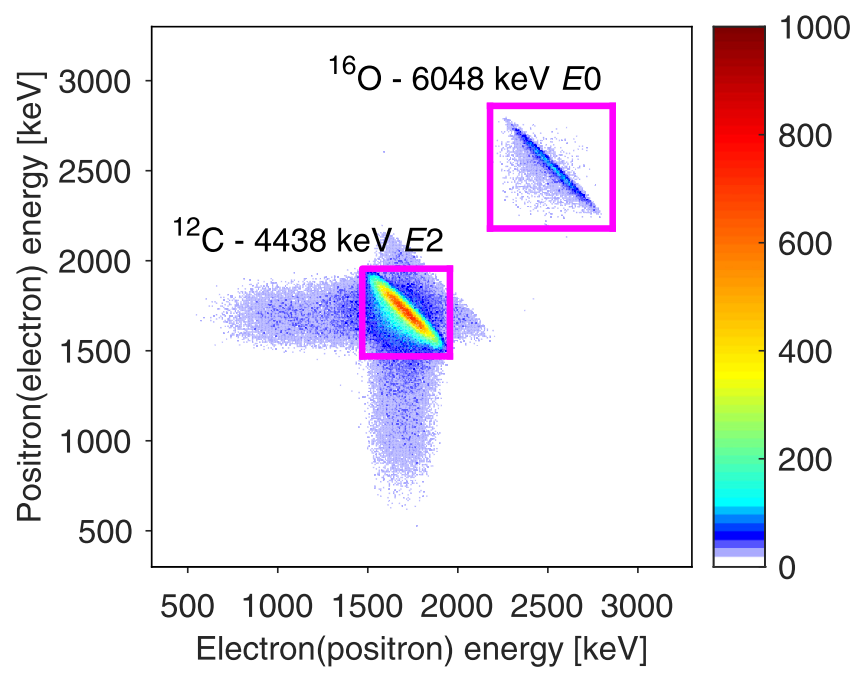

FIG. 4. Energy vs. energy from a ${ }^{12} \mathrm{C}$ pair conversion measurement, showing the $4.44 \mathrm{MeV}$ and $6.05 \mathrm{MeV}$ transitions in ${ }^{12} \mathrm{C}$ and ${ }^{16} \mathrm{O}$, respectively. Note that the pair distribution for the $4.44 \mathrm{MeV}$ transition is broadened due to the Doppler effect caused by decay from moving target recoils. The solid lines indicate the bounds of the momentum window, calculated as described in Ref. [26]. The color scale indicates the number of counts.

More specifically, the electron and positron share the available transition energy, less the energy consumed in the creation of two electron masses, $2 m_{0} c^{2}$, according to the doubledifferential pair-emission probability. The double differential is defined as a function of positron energy, $E_{+}$, and separation angle of the pair, $\theta_{s}$, and depends on the transition energy and multipolarity. Figure 5 illustrates the kinematics of a pair emission in the spectrometer frame of reference.

In the present work, the double differential pair emission probability was calculated within the Born approximation with Coulomb correction, which will be explained in the following. Comparison of the distributions calculated with the Born approximation integrated over $\theta_{s}$, and single differential values for finite size calculations from Refs. [28,29], showed that the agreement was better than $1 \%$ for $Z=6$ when

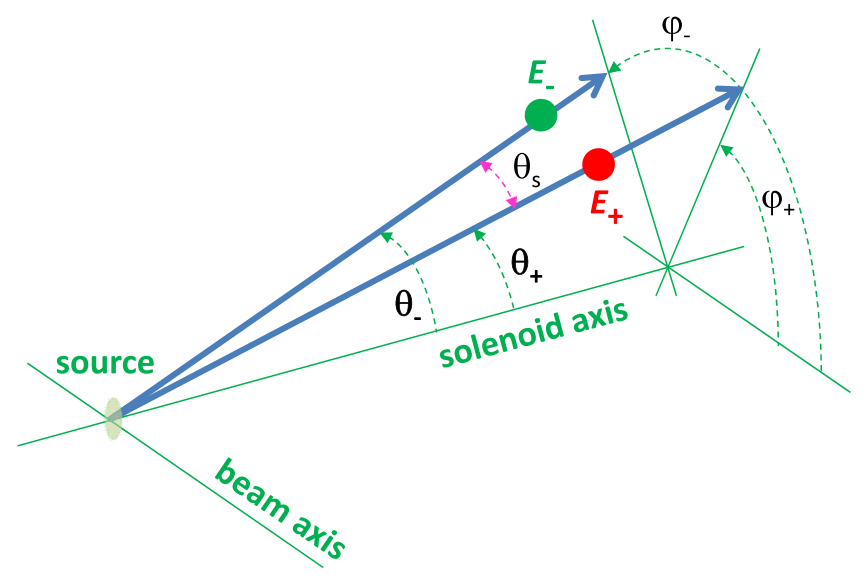

FIG. 5. Pair emission in the spectrometer frame of reference. The intersection of the beam axis and spectrometer symmetry axis defines the origin of the coordinate system. 
$E_{-} \approx E_{+}$. Hence, the Born approximation was considered satisfactory for the ${ }^{12} \mathrm{C}$ pair emission simulations. The double differential probability distribution for $E 0$ pair transitions is determined by [30]

$$
\begin{aligned}
\frac{d^{2} \Omega_{\pi}(E 0)}{d E_{+} d \cos \theta_{s}}= & p_{+} p_{-}\left(W_{+} W_{-}-m_{0}^{2} c^{4}+p_{+} p_{-} c^{2} \cos \theta_{s}\right), \quad \text { transition [31] by } \\
\frac{d^{2} \alpha_{\pi}(E L)}{d E_{+} d \cos \theta_{s}}= & \left(\frac{2 \alpha}{\pi(L+1)}\right)\left(\frac{p_{+} p_{-}}{q}\right) \frac{(q / \omega)^{2 L-1}}{\left(\omega^{2}-q^{2}\right)^{2}} \times\left[(2 L+1)\left(W_{+} W_{-}+1-\frac{p_{+} p_{-}}{3} \cos \theta_{s}\right)\right. \\
& \left.+L\left(\frac{q^{2}}{\omega^{2}}-2\right)\left(W_{+} W_{-}-1+p_{+} p_{-} \cos \theta_{s}\right)+\frac{1}{3}(L-1) p_{+} p_{-}\left(\frac{3}{q^{2}}\left(p_{-}+p_{+} \cos \theta_{s}\right)\left(p_{+}+p_{-} \cos \theta_{s}\right)-\cos \theta_{s}\right)\right]
\end{aligned}
$$

where $p_{ \pm}$denote momenta and $W_{ \pm}=E_{ \pm}+m_{0} c^{2}$ the total energies for electrons $(-)$ and positrons $(+)$. For higher electric multipoles, $E L$, the double differential distribution is given in terms of the pair conversion coefficient of the where $\alpha$ is the fine structure constant, $q$ is the magnitude of the quantization vector, $\vec{q}=\vec{p}_{+}+\vec{p}_{-}$, and $\omega$ denotes the transition energy. It is important to note that in Eq. (8), $\hbar=m_{0}=c=1$, so all energies are in terms of $m_{0} c^{2}$ and $p=\sqrt{W^{2}-1}$.

The evaluation of the pair transmission efficiency was performed using Monte Carlo simulations, by first simulating emission, and then transmission through the spectrometer. Pair emission was then sampled from the double differential probability distribution of Eq. (7) for the $E 0$ transition, and according to Eq. (8) for the $E 2$ transition. The distributions were corrected for Coulomb distortion of the emitted electron and positron energies, by multiplication with a correction factor as a function of positron energy. The Coulomb correction factor was estimated as described in Appendix H in Ref. [32]

$$
F=\frac{\left(2 \pi B_{+}\right)\left(2 \pi B_{-}\right)}{\left(\exp \left(2 \pi B_{+}\right)-1\right)\left(1-\exp \left(-2 \pi B_{-}\right)\right)},
$$

where $B_{ \pm}$denotes the relativistic Sommerfeld parameter $\left(Z \alpha E_{ \pm}\right) / p_{ \pm}$. The Coulomb correction, which also depends on the energy budget of the pair, is applied by multiplication with the distributions provided in Eqs. (7) and (8). Double differential distributions calculated for the $3.22 \mathrm{MeV} E 2$ and the 7.65 MeV E0 transitions from the Hoyle state in ${ }^{12} \mathrm{C}$ are shown in Figs. 6(a) and 6(b), respectively. Pairs emitted in the $4.44 \mathrm{MeV} E 2$ transition are distributed in a similar fashion as shown in Fig. 6(a), but with a different energy range. Since the $4.44 \mathrm{MeV} \mathrm{E2}$ transition originates from the $2_{1}^{+}$state of ${ }^{12} \mathrm{C}$, it is necessary to account for alignment of the nuclear spin states induced by the reaction and the effects on the corresponding pair emission distribution. The alignment correction is evaluated by using the distribution coefficients, $A_{2}$ and $A_{4}$, of the Legendre polynomials associated with the $\gamma$-ray angular distribution of the transition

$$
W_{\gamma}\left(\theta_{\mathrm{lab}}\right)=A_{0}+A_{2} P_{2}\left(\cos \theta_{\mathrm{lab}}\right)+A_{4} P_{4}\left(\cos \theta_{\mathrm{lab}}\right),
$$

where $P_{v}$ denotes the Legendre polynomial of order $v$, and $\theta_{\text {lab }}$ is the $\gamma$-ray emission angle in the laboratory relative to the beam axis. The procedure for applying these coefficients to correct Eq. (8) for alignment is explained in Refs. [33,34].

Trajectories of electrons and positrons emitted from the target were simulated by solving the relativistic equations of motion with the fourth order Runge-Kutta method. The equations were solved in a realistic magnetic-field profile for the solenoid calculated with Poisson Superfish [35], and the particle trajectories were projected within a detailed specification of the spectrometer geometry in the spectrometer frame of reference. A trajectory calculation was terminated if the corresponding particle struck the surface of the absorber system or the inner bore. If both the electron and positron reached a $\mathrm{Si}(\mathrm{Li})$ segment, the event was registered as successful and all the parameters were stored. The pair-transmission efficiency was ultimately found by the ratio of pairs reaching two separate detector segments versus the number of emitted pairs. Transmitted and detected events of the emitted 3.22 MeV E2 and 7.65 MeV E0 pair transitions are shown in Figs. 6(c) and

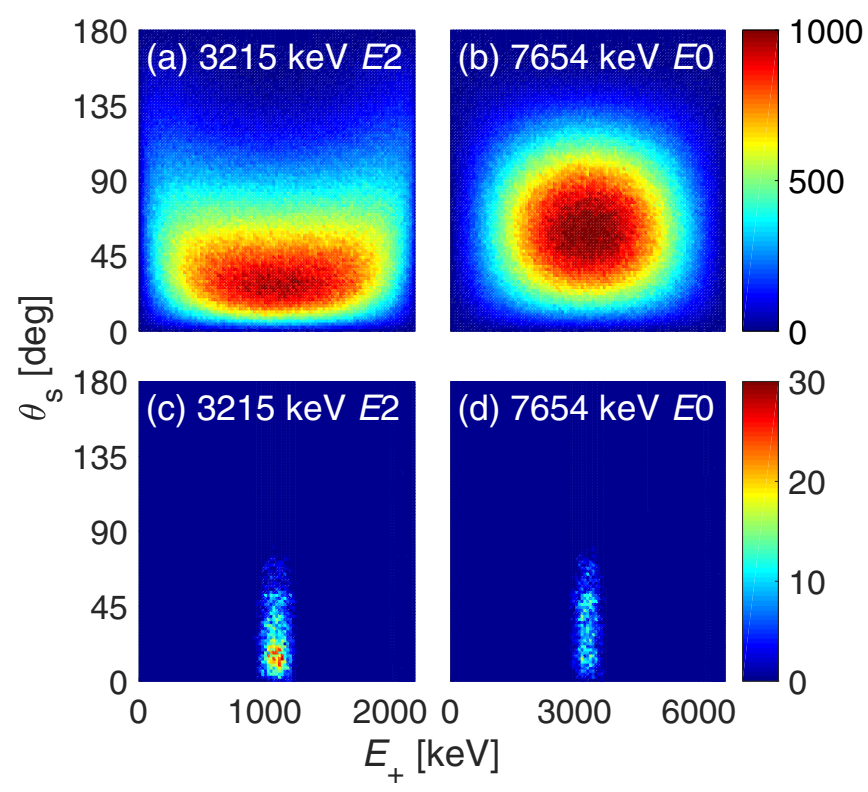

FIG. 6. Monte Carlo simulations of pair emission and transmission. (a) and (b) Double differential pair emission distributions for the transitions de-exciting the Hoyle state. The maximum emission probabilities are for $E_{-} \approx E_{+}$, and $\theta_{s}=30^{\circ}$ and $60^{\circ}$ for the $E 2$ and $E 0$ transitions, respectively. (c) and (d) Detected pairs following emission according to the distributions in (a) and (b) and allowing for transmission through the spectrometer. 
6(d), respectively. A potential cause of systematic uncertainty in the transmission efficiency would be from the use of the Born approximation with Coulomb correction, as opposed to applying calculations for extended nuclei. However, as mentioned above, the Born approximation differs by less than $1 \%$ from the extended nuclei calculations performed for the low- $Z{ }^{12} \mathrm{C}$ nucleus, and no systematic uncertainties were assumed for the simulated transmission efficiency in the present work.

The availability of sources for determining pair detection efficiencies, and even singles conversion electron detection efficiencies, is very limited. Consequently, the intrinsic detector efficiency was deduced from Monte Carlo simulations performed with the penelope simulation tool [36].

Simulated spectra have previously been compared to ${ }^{133} \mathrm{Ba}$ and ${ }^{56} \mathrm{Co}$ conversion electron measurements [37], with the conclusion that penelope is reliable for the electron and positron energies relevant for the current work. Simulations of transmitted monoenergetic electrons and positrons between $0.1-8 \mathrm{MeV}$ were used as input for interaction simulations in conjunction with detailed geometry and material specifications of the detector array and spectrometer. The input parameters include realistic distributions of incident electron and positron angles, which are important for consideration of back-scattering. After folding in a resolution of $5 \mathrm{keV}$ to the resulting response spectra, the intrinsic efficiency was deduced from the ratio of counts in the full energy peak versus the total number of counts in the spectrum. The intrinsic efficiencies obtained from the simulations are shown in Fig. 7. Note that the positron efficiency is in general lower than the corresponding electron efficiency. This is due to the fact that the positron-response spectra have an additional component above the full (kinetic) energy peak caused by energy deposition by annihilation radiation (converted from the rest mass). Hence, the ratio of counts in the full energy peak versus total counts is lower for positrons than it is for electrons. The interaction cross sections of electrons and positrons in matter are otherwise essentially identical for the incident energies relevant to the pair measurements in the present work.

\section{Experimental conditions}

The 4.44 MeV $2_{1}^{+}$and $7.65 \mathrm{MeV} 0_{2}^{+}$levels in ${ }^{12} \mathrm{C}$ were populated by using the ${ }^{12} \mathrm{C}\left(p, p^{\prime}\right)$ reaction at $10.5 \mathrm{MeV}$ proton energy, which is a resonant bombarding energy for population of the Hoyle state [38]. Target foils of $1 \mathrm{mg} / \mathrm{cm}^{2}$ and $2 \times$ $1 \mathrm{mg} / \mathrm{cm}^{2}$ natural carbon containing $98.9 \%{ }^{12} \mathrm{C}$ and $1.1 \%{ }^{13} \mathrm{C}$ were used. The beam intensity varied between $0.5-1.0 \mu \mathrm{A}$, but was mostly stable around 500-600 nA. For the chosen target and beam energy, the cross sections for populating the $4.44 \mathrm{MeV}$ and $7.65 \mathrm{MeV}$ levels are reported to be $\sigma_{4.44}=291$ $\mathrm{mb}$ [39] and $\sigma_{7.65}=86.5 \mathrm{mb}$ [38], respectively. The average energy losses of $10.5 \mathrm{MeV}$ protons in the full thicknesses of the $1 \mathrm{mg} / \mathrm{cm}^{2}$ and $2 \times 1 \mathrm{mg} / \mathrm{cm}^{2}$ target foils positioned at $45^{\circ}$ relative to the beam are $56 \mathrm{keV}$ and $110 \mathrm{keV}$ [40], respectively. A simple reaction rate calculation with $10.5 \mathrm{MeV}$ monoenergetic protons and a beam intensity of $500 \mathrm{nA}$ impinging on a $1 \mathrm{mg} / \mathrm{cm}^{2}$ target, yields rates of $r_{4.44}=6.94 \times$

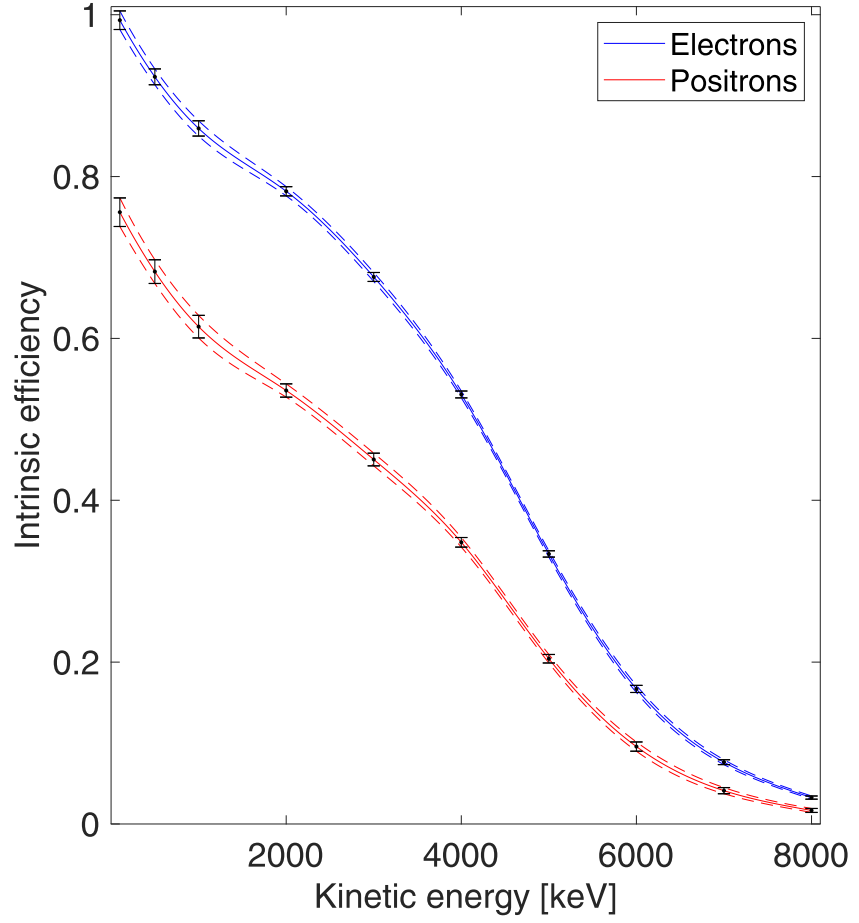

FIG. 7. Intrinsic electron (blue/upper line) and positron (red/lower line) detection efficiencies of the Miel $\mathrm{Si}(\mathrm{Li})$ array, deduced from Monte Carlo simulations (data points). The error bars are defined as $3 \sigma$ of the statistical uncertainties. The solid lines represent interpolations between data points, and the dashed lines indicate the uncertainties.

$10^{7} \mathrm{~s}^{-1}$ and $r_{7.65}=2.06 \times 10^{7} \mathrm{~s}^{-1}$ for population of the two excited states. By taking into account the relevant conversion coefficients, branching ratios, and spectrometer transmission, the rates of pair constituents striking different detector segments in coincidence were deduced. The deduced rates are 0.4 pairs $/ \mathrm{min}$ for the $3.22 \mathrm{MeV} \mathrm{E} 2$ transition, 4860 pairs $/ \mathrm{min}$ for the $4.44 \mathrm{MeV} E 2$ transition and 5.5 pairs/min for the 7.65 MeV E0 transition. These rates were calculated using optimum magnetic fields for transmission of pairs from the transitions, which are $0.20 \mathrm{~T}, 0.28 \mathrm{~T}$, and $0.49 \mathrm{~T}$, respectively. Furthermore, the target contained a small fraction of ${ }^{16} \mathrm{O}$, which allowed the $6.05 \mathrm{MeV} \mathrm{E0}$ pair transition from the $0_{2}^{+}$ state to be sampled and conveniently used for energy calibration in conjunction with the strong $4.44 \mathrm{MeV}$ transition in ${ }^{12} \mathrm{C}$. The optimum magnetic field for measuring the $6.05 \mathrm{MeV}$ E0 transition was $0.40 \mathrm{~T}$. The magnetic field of the solenoid was stepped through the four discrete optimum field settings over several repeated cycles during each run. Each cycle had a duration of about 30 minutes, and the time spent at each magnetic field was controlled by the integrated beam current on the target. The amount of time allocated to each field was determined by the expected intensity of the transitions, with more time allocated to weaker transitions. In the present measurements, $68 \%, 3 \%, 3 \%$, and $26 \%$ of a cycle was allocated to the 3.22 MeV, 4.44 MeV, 6.05 MeV, and 7.65 MeV transitions, respectively. 


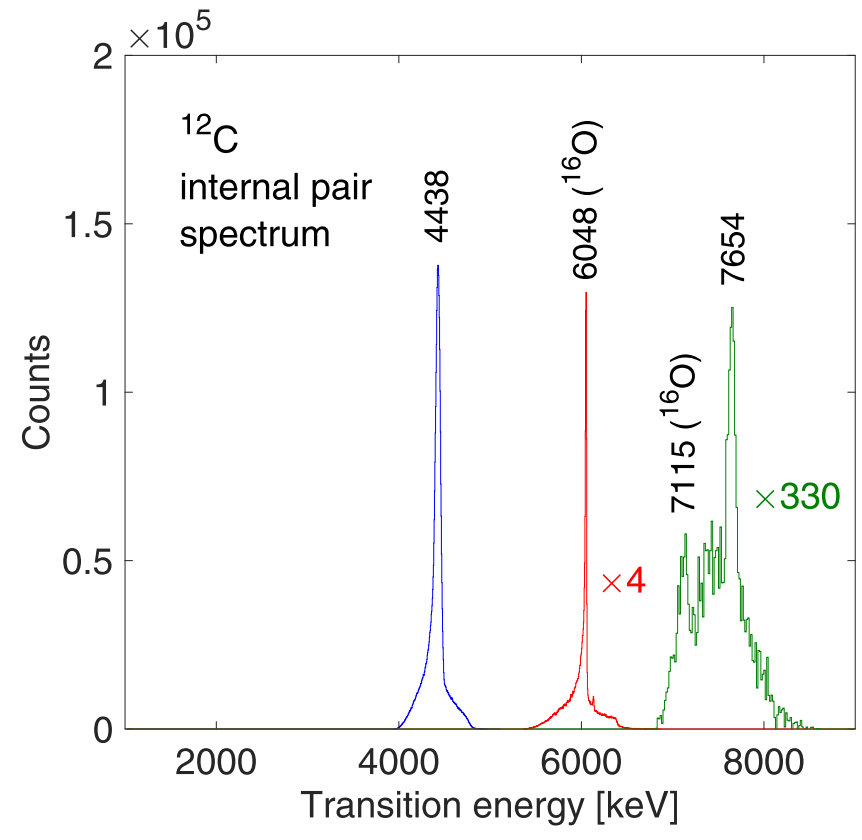

FIG. 8. The summed pair spectrum of the three ${ }^{12} \mathrm{C}$ experimental runs. The transitions are normalized to the peak area of the $4.44 \mathrm{MeV}$ $\gamma$-ray transition measured by the monitor detector. Note that the $6048 \mathrm{keV}$ and $7654 \mathrm{keV}$ lines have been scaled up for visualization purposes.

\section{RESULTS}

Four transitions were sampled during the pair measurements of the present work. They were the $4.44 \mathrm{MeV}_{1}^{+} \rightarrow$ $0_{1}^{+}, 3.22 \mathrm{MeV}_{2}^{+} \rightarrow 2_{1}^{+}$, and $7.65 \mathrm{MeV}_{2}^{+} \rightarrow 0_{1}^{+}$transitions in ${ }^{12} \mathrm{C}$, and the $6.05 \mathrm{MeV} 0_{2}^{+} \rightarrow 0_{1}^{+}$transition in ${ }^{16} \mathrm{O}$. An initial objective was to detect the $3.22 \mathrm{MeV} E 2$ pair transition from the Hoyle state, however, this turned out to be too ambitious as a large background rendered the observation of this weak transition impossible. Instead, the focus turned to the $4.44 \mathrm{MeV}$ and the $7.65 \mathrm{MeV}$ transitions, which were clearly visible. These two pair transitions in ${ }^{12} \mathrm{C}$, as well as the ${ }^{16} \mathrm{O}$ line used for energy calibration, are shown in Fig. 8. Note that the spectrum has been shifted up in energy by $2 m_{0} c^{2}=1022 \mathrm{keV}$ to reflect the transition energy. The spectrum in Fig. 8 corresponds to 9 days of beam on target, from three experimental runs. To account for sampling time and beam intensity, the individual spectra were normalized to the peak area of the $4.44 \mathrm{MeV} \gamma$-ray transition measured by the monitor detector before summation. Furthermore, the spectra have been random subtracted by applying gates on prompt and random time differences.

Since the 4.44 MeV E2 transition originates from the $2_{1}^{+}$ state, the pair emission distribution for the transmission efficiency calculation had to be corrected for nuclear alignment effects. In order to obtain the distribution coefficients needed for the correction, the $\gamma$-ray intensities of the $4.44 \mathrm{MeV}$ transition were measured at $\theta_{\mathrm{lab}}=20^{\circ}-160^{\circ}$ in $10^{\circ}$ steps, using a HPGe detector with a crystal size of $81 \mathrm{~mm} \times 54 \mathrm{~mm}$ (length $\times$ diameter) positioned $41.5 \mathrm{~cm}$ away from the target. The attenuation factors for this setup were found to be close

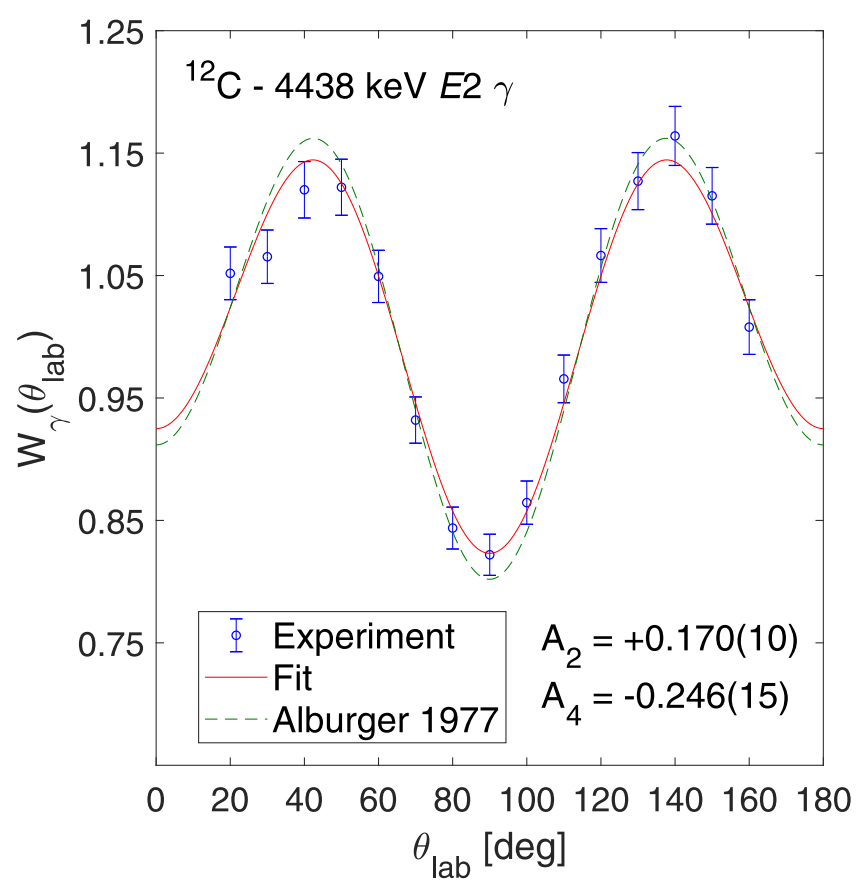

FIG. 9. The angular distribution of $\gamma$ rays from the $2_{1}^{+} \rightarrow 0_{1}^{+}$ transition in ${ }^{12} \mathrm{C}$. The results are in good agreement with the previous measurement performed by Alburger [23].

to unity. For these measurements, a $1 \mathrm{mg} / \mathrm{cm}^{2}$ thick natural carbon target was used, and the $2_{1}^{+}$state was populated by the ${ }^{12} \mathrm{C}\left(p, p^{\prime}\right)$ reaction at $10.5 \mathrm{MeV}$. The resulting angular distribution is shown with fitted distribution coefficients in Fig. 9, and corresponds very well with the one measured by Alburger in 1977 [23]. By comparing Monte Carlo simulations for pair transitions from unaligned and aligned cases of the 4.44 $\mathrm{MeV}$ state, a $7.45 \%$ reduction in transmission efficiency was revealed for the aligned case with the measured distribution coefficients.

The proton population ratio, $N_{p}\left(2_{1}^{+}\right) / N_{p}\left(0_{2}^{+}\right)$, is also needed to extract $\Gamma_{\pi}^{E 0} / \Gamma$ from the pair measurements according to the method described in Sec. II. For this reason, scattering measurements of ${ }^{12} \mathrm{C}\left(p, p^{\prime}\right)$ were carried out using the ANU BALiN double sided silicon strip detector array [41-43]. The proton scattering distributions of the $2_{1}^{+}$and $\mathrm{O}_{2}^{+}$states were measured simultaneously for scattering angles between $20^{\circ}-160^{\circ}$. Measurements were performed using both a $50 \mu \mathrm{g} / \mathrm{cm}^{2}$ and the same $1 \mathrm{mg} / \mathrm{cm}^{2}$ thick ${ }^{12} \mathrm{C}$ target foil used in the pair conversion measurements. The $50 \mu \mathrm{g} / \mathrm{cm}^{2}$ thick target was bombarded over several runs with proton beams of energies ranging between $10.4-10.7 \mathrm{MeV}$, to obtain the angular distributions as a function of proton energy with little effect of energy loss in the target. The $1 \mathrm{mg} / \mathrm{cm}^{2}$ thick target was bombarded with $10.5 \mathrm{MeV}$ protons to obtain the proton angular distributions under the same conditions as in the ${ }^{12} \mathrm{C}$ pair measurements of the present work. The angular distributions will be discussed in detail in a separate paper [44].

Angular distribution functions were fitted to the data, and the ratio of the integrals over the full solid angle were 


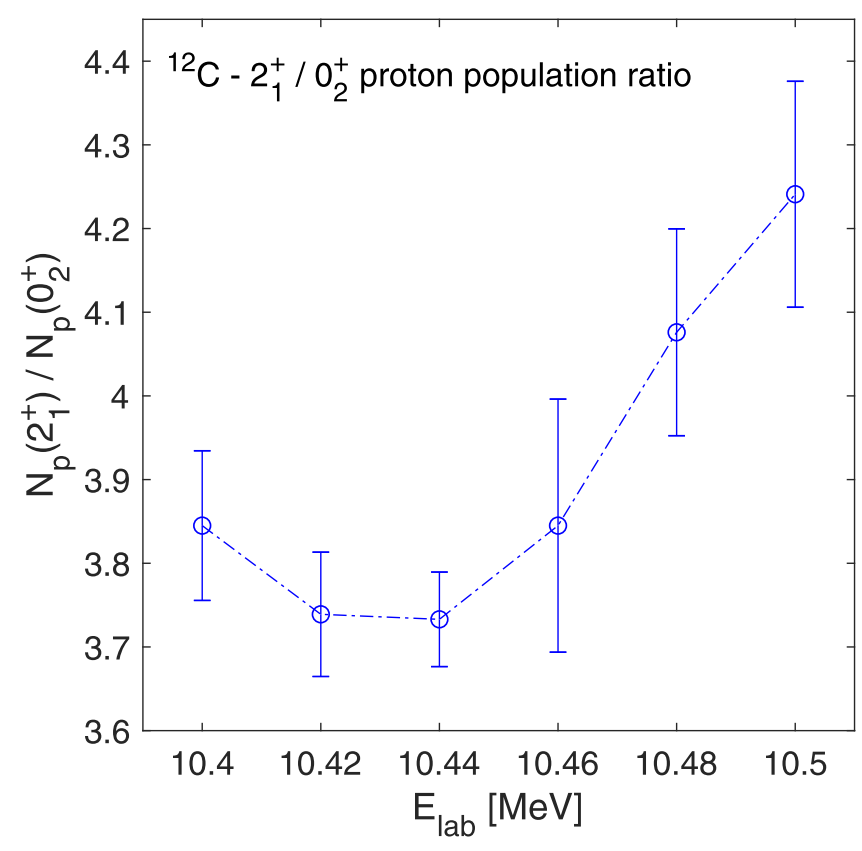

FIG. 10. Proton population ratio of the $2_{1}^{+}$and $0_{2}^{+}$states in ${ }^{12} \mathrm{C}$ as a function of proton beam energy. The dashed line represents a linear interpolation between the data points.

used to deduce the proton population ratio of the $2_{1}^{+}$and $0_{2}^{+}$states. The $50 \mu \mathrm{g} / \mathrm{cm}^{2}$ target measurements provided $N_{p}\left(2_{1}^{+}\right) / N_{p}\left(0_{2}^{+}\right)$as a function of proton energy, which are shown in Fig. 10 for energies relevant to the present work. By averaging the population ratio over the proton energy loss in the targets used in the pair measurements, proton population ratios of $N_{p}\left(2_{1}^{+}\right) / N_{p}\left(0_{2}^{+}\right)=3.97(12)$ and $3.89(10)$ were obtained for the $1 \mathrm{mg} / \mathrm{cm}^{2}$ and $2 \times 1 \mathrm{mg} / \mathrm{cm}^{2}$ thick targets, respectively. The corresponding ratio obtained from the proton scattering measurement with the $1 \mathrm{mg} / \mathrm{cm}^{2}$ thick target yields $N_{p}\left(2_{1}^{+}\right) / N_{p}\left(0_{2}^{+}\right)=3.96(4)$, in excellent agreement with the averaged value. A weighted mean of $N_{p}\left(2_{1}^{+}\right) / N_{p}\left(0_{2}^{+}\right)=$ $3.961(38)$ was adopted for the $1 \mathrm{mg} / \mathrm{cm}^{2}$ target. These results are consistent with the previous value of $N_{p}\left(2_{1}^{+}\right) / N_{p}\left(0_{2}^{+}\right)=$ 3.74(18) from Alburger [23], which was deduced for a 3.5 $\mathrm{mg} / \mathrm{cm}^{2}$ thick target.

The E0 pair branching ratio was deduced separately for the three pair measurements according to Eq. (6), using the measured pair count ratios listed in the third column of Table I, and the relevant population ratio (second column), pair detection efficiencies, and the theoretical pair conversion coefficient $[28,45]$. The efficiencies and conversion coefficient that were used in all three calculations are summarized in Table II. The resulting $\Gamma_{\pi}^{E 0} / \Gamma$ values are listed in the fourth column of Table I. An average E0 pair decay branching ratio of $\Gamma_{\pi}^{E 0} / \Gamma=8.2(5) \times 10^{-6}$ was found using AveTools [46], which utilizes three different methodologies to evaluate the average. These are the limitation of relative statistical weight, normalized residual method, and the Rajeval technique, which are explained in detail in Ref. [47]. The three methods returned the same average value and uncertainty. A summary of the previous, current, and a weighted average of the $E 0$ pair
TABLE I. The experimental quantities used to deduce the $E 0$ pair branching ratio of the Hoyle state, and the resulting values. The weighted average was obtained using AveTools [46].

\begin{tabular}{llcl}
\hline \hline Run & $N_{p}\left(2_{1}^{+}\right) / N_{p}\left(0_{2}^{+}\right)$ & $N_{\pi}^{E 0} / N_{\pi}^{E 2} \times 10^{4}$ & $\Gamma_{\pi}^{E 0} / \Gamma \times 10^{6}$ \\
\hline 1 & $3.89(10)$ & $6.98(68)$ & $8.19(89)$ \\
2 & $3.961(38)$ & $6.81(43)$ & $8.14(62)$ \\
3 & $3.961(38)$ & $6.85(103)$ & $8.19(128)$ \\
& & Weighted average: & $8.2(5)$ \\
\hline \hline
\end{tabular}

branching ratios is provided in Fig. 11. The weighted average in Fig. 11 was also found using AveTools.

\section{DISCUSSION}

As can be seen in Fig. 11, there are four previous published values for the $E 0$ pair decay branching ratio of the Hoyle state. The results of Ajzenberg et al. [20] and Obst et al. [21] come from measurements of the neutron population ratio, $N_{n}\left(2_{1}^{+}\right) / N_{n}\left(0_{2}^{+}\right)$, in the reaction ${ }^{9} \mathrm{Be}(\alpha, n){ }^{12} \mathrm{C}$ at $E_{\alpha}=5.81$ $\mathrm{MeV}$, while their results for the $E 0$ pair branching ratio of the Hoyle state are both based on the pair intensity ratio, $N_{\pi}^{E 0} / N_{\pi}^{E 2}$, measured by Alburger in 1960 [19] under the same experimental conditions. Robertson et al. [22] applied an independent and direct approach to deduce $\Gamma_{\pi}^{E 0} / \Gamma$, by measuring the ratio of protons in coincidence with a $7.65 \mathrm{MeV}$ pair transition over the singles proton rate $N_{p, \pi}^{E 0}\left(0_{2}^{+}\right) / N_{p}^{\text {tot }}\left(0_{2}^{+}\right)$in a ${ }^{12} \mathrm{C}\left(p, p^{\prime}\right)$ experiment at $E_{p}=10.56 \mathrm{MeV}$. The pair transitions were detected with a plastic scintillator detector covering nearly the full solid angle around the target, thus providing close to $100 \%$ pair detection efficiency. However, due to the nature of the experimental setup, a number of corrections and uncertainties had to be considered in their analysis. In 1977, Alburger performed a pair intensity ratio measurement using the ${ }^{12} \mathrm{C}\left(p, p^{\prime}\right)$ reaction at $10.5 \mathrm{MeV}$ [23]. The advantages of this approach are the resonant reaction for populating the Hoyle state, and the relative ease of measuring population ratios of protons as compared to neutrons. Alburger then deduced the $E 0$ pair branching ratio according to the method described in Sec. II. The same approach was adopted in the present work using data measured with the ANU Super-e spectrometer. The great improvement in resolving power of the present measurements, as compared to Alburger [23], is demonstrated in Fig. 12. The $E 0$ pair branching ratio deduced from the present measurements, $\Gamma_{\pi}^{E 0} / \Gamma=8.2(5) \times 10^{-6}$, agrees with that deduced by Alburger, $\Gamma_{\pi}^{E 0} / \Gamma=7.1(8) \times$ $10^{-6}$, within the uncertainties. We recommend a weighted average of the previous and current measurements of the $E 0$ pair decay branching ratio, $\Gamma_{\pi}^{E 0} / \Gamma=7.6(4) \times 10^{-6}$, for

TABLE II. The detection efficiencies and conversion coefficient used to deduce the $E 0$ pair branching ratio of the Hoyle state. The pair conversion coefficient was obtained from BrIcc [28,45].

\begin{tabular}{lcc}
\hline \hline$\epsilon_{\pi}^{E 2}$ & $\epsilon_{\pi}^{E 0}$ & $\alpha_{\pi}$ \\
\hline $3.96(10) \times 10^{-4}$ & $1.73(5) \times 10^{-4}$ & $1.32(2) \times 10^{-3}$ \\
\hline \hline
\end{tabular}




\section{Ajzenberg et al. (1960) \\ Obst et al. (1972) \\ Robertson et al. (1977) \\ Alburger (1977) \\ Present work \\ Weighted average: 7.6(4)

$$
\begin{aligned}
& \begin{array}{llllll}
4 & 5 & 6 & 7 & 8 & 9
\end{array} \\
& \Gamma_{\pi}(E O) / \Gamma\left[10^{-6}\right]
\end{aligned}
$$

FIG. 11. Previous, current, and weighted average values of $\Gamma_{\pi}^{E 0} / \Gamma$. Further information about the previous measurements can be found in Refs. [20-23] (listed in chronological order).

calculation of the radiative width of the Hoyle state. As a result, the present work reduces the uncertainty of the $E 0$ pair branching ratio to $5 \%$ and increases its value by $14 \%$ compared to the one adopted in the recent review by Freer and Fynbo [6], $\Gamma_{\pi}^{E 0} / \Gamma=6.7(6) \times 10^{-6}$. The new value of $\Gamma_{\pi}^{E 0} / \Gamma$ provides a radiative width of $\Gamma_{\text {rad }}=3.28(20) \mathrm{meV}$ when combined with the radiative branching ratio and $E 0$ decay width reported in Ref. [6], i.e., $\Gamma_{\text {rad }} / \Gamma=4.03(10) \times$ $10^{-4}[11-18]$ and $\Gamma_{\pi}^{E 0}=62.3(20) \mu \mathrm{eV}$ [24]. Compared to the previously adopted value of the radiative width of the Hoyle state, $\Gamma_{\text {rad }}=3.7(4) \mathrm{meV}$ [6], the new result for the width agrees within the error bars, but is $11 \%$ smaller and has an uncertainty of $6.1 \%$ as compared to $10 \%$.

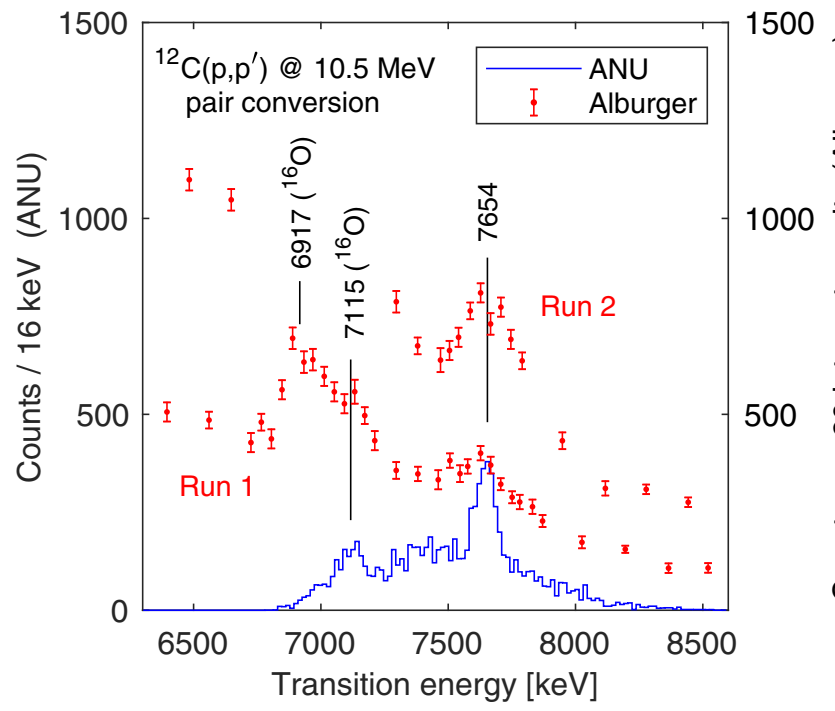

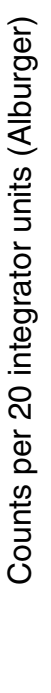

FIG. 12. A comparison of the $7.65 \mathrm{MeV} E 0$ pair spectra from the present measurement and Alburger's 1977 experiment [23] to show the improved resolution and suppression of ${ }^{16} \mathrm{O}$ peaks. The energy region containing the ${ }^{16} \mathrm{O}$ peaks was excluded in Alburger's second run to save time.

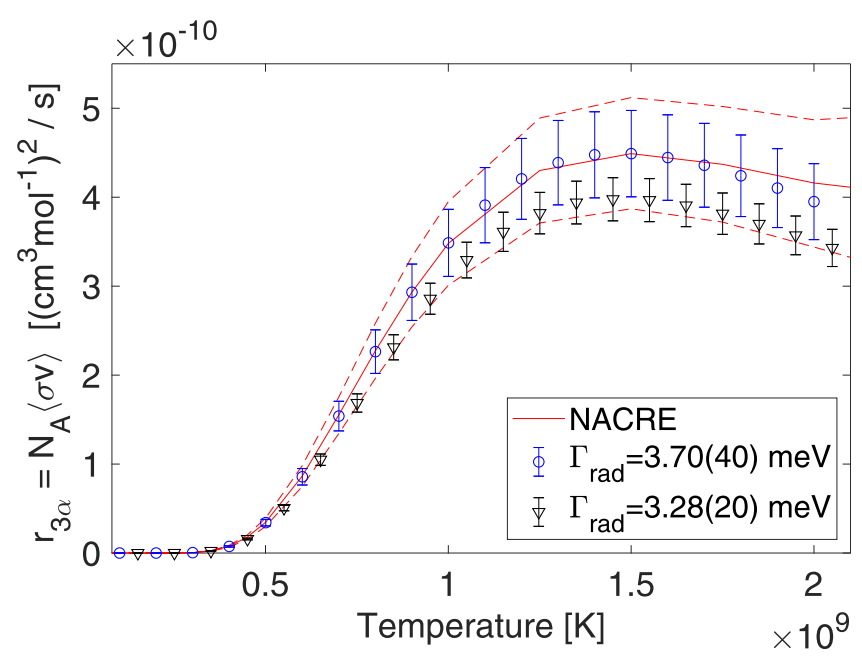

FIG. 13. The $3 \alpha$ reaction rate calculated within the temperature range of helium burning red giant stars using the NACRE library value [48] (solid red line with dashed lines indicating the range of uncertainty), previous recommended value (blue circles), and new recommended value (black triangles) of the radiative width of the Hoyle state.

Plots of the estimated $3 \alpha$ reaction rates, $r_{3 \alpha}$, using the previous and current radiative widths are provided in Fig. 13. The figure also includes rates calculated with the standard NACRE library value [48]. The reaction rates agree well within the uncertainties, and it is clear that the new value on $\Gamma_{\text {rad }}$ would not significantly change our astrophysical models and predictions. However, the reduced uncertainty will constrain possible scenarios and outcomes of the calculations, and facilitates advances in the research on stellar evolution and element synthesis in the universe. A major implication of $r_{3 \alpha}$ is its effect on the carbon-to-oxygen abundance ratio at the end of the helium burning phase of stars, in which the $3 \alpha$ process and ${ }^{12} \mathrm{C}(\alpha, \gamma){ }^{16} \mathrm{O}$ reaction compete for the available $\alpha$ particles, with the latter reaction also feeding on the available ${ }^{12} \mathrm{C}$ nuclei. The carbon-to-oxygen abundance ratio is important for later stages of stellar evolution, and the rates of production and consumption of ${ }^{12} \mathrm{C}$ are therefore important input parameters in astrophysical calculations.

A recent measurement [49] of the radiative branching ratio, $\Gamma_{\text {rad }} / \Gamma$, suggests a value that is substantially higher than the currently adopted ratio used in this work. Combining this recent result with the present measurement on $\Gamma_{\pi}^{E 0} / \Gamma$, results in a large increase of the radiative width as compared to the adopted value. This increase would have a significant impact on astrophysical calculations, and it is crucial to address the discrepancy observed for $\Gamma_{\text {rad }} / \Gamma$.

A new approach to determine the radiative width from a direct measurement of the ratio of the pair transitions deexciting the Hoyle state, $\Gamma_{\pi}^{E 2} / \Gamma_{\pi}^{E 0}$, has been developed [27]. However, the success of this new method requires a 20 times reduction in the background currently observed in vicinity of the $3.22 \mathrm{MeV} E 2$ pair peak. If this can be done, this approach has the potential to provide an independent measurement and settle the discrepancy for $\Gamma_{\text {rad }}$. 


\section{ACKNOWLEDGMENTS}

The project was supported by the Australian Research Council Discovery Grants No. DP140102986, No. DP170101673, and No. DP170102423. Operation of the ANU Heavy Ion Accelerator Facility is supported by the NCRIS HIA capability. The support from technical staff for the de- velopment of the pair spectrometer, as well as during the long experimental runs, is greatly appreciated. This work was partially supported by the International Joint Research Promotion Program of Osaka University and JSPS KAKENHI Grant No. JP 17H02893, the Natural Sciences and Engineering Research Council of Canada, the National Research Foundation (NRF), South Africa, under Grants No. 93533 and No. 118645.
[1] G. Audi, F. G. Kondev, M. Wang, W. J. Huang, and S. Naimi, Chin. Phys. C 41, 030001 (2017).

[2] E. E. Salpeter, Astrophys. J. 115, 326 (1952).

[3] F. Hoyle, Astrophys. J. Suppl. Ser. Sup 1, 121 (1953).

[4] D. N. F. Dunbar, R. E. Pixley, W. A. Wenzel, and W. Whaling, Phys. Rev. 92, 649 (1953).

[5] C. W. Cook, W. A. Fowler, C. C. Lauritsen, and T. Lauritsen, Phys. Rev. 107, 508 (1957).

[6] M. Freer and H. O. U. Fynbo, Prog. Part. Nucl. Phys. 78, 1 (2014).

[7] D. Dell'Aquila, I. Lombardo, G. Verde, M. Vigilante, L. Acosta, C. Agodi, F. Cappuzzello, D. Carbone, M. Cavallaro, S. Cherubini, A. Cvetinovic, G. D'Agata, L. Francalanza, G. L. Guardo, M. Gulino, I. Indelicato, M. La Cognata, L. Lamia, A. Ordine, R. G. Pizzone, S. M. R. Puglia, G. G. Rapisarda, S. Romano, G. Santagati, R. Sparta, G. Spadaccini, C. Spitaleri, and A. Tumino, Phys. Rev. Lett. 119, 132501 (2017).

[8] R. Smith, Tz. Kokalova, C. Wheldon, J. E. Bishop, M. Freer, N. Curtis, and D. J. Parker, Phys. Rev. Lett. 119, 132502 (2017).

[9] T. K. Rana, S. Bhattacharya, C. Bhattacharya, S. Manna, S. Kundu, K. Banerjee, R. Pandey, P. Roy, A. Dhal, G. Mukherjee, V. Srivastava, A. Dey, A. Chaudhuri, T. K. Ghosh, A. Sen, Md. A. Asgar, T. Roy, J. K. Sahoo, J. K. Meena, A. K. Saha, R. M. Saha, M. Sinha, and A. Roy, Phys. Lett. B 793, 130 (2019).

[10] C. E. Rolfs and W. S. Rodney, Cauldrons in the Cosmos (The University of Chicago Press, Chicago, 1988).

[11] D. E. Alburger, Phys. Rev. 124, 193 (1961).

[12] P. A. Seeger and R. W. Kavanagh, Nucl. Phys. 46, 577 (1963).

[13] I. Hall and N. W. Tanner, Nucl. Phys. 53, 673 (1964).

[14] D. Chamberlin, D. Bodansky, W. W. Jacobs, and D. L. Oberg, Phys. Rev. C 9, 69 (1974).

[15] C. N. Davids, R. C. Pardo, and A. W. Obst, Phys. Rev. C 11, 2063 (1975)

[16] H.-B. Mak, H. C. Evans, G. T. Ewan, A. B. McDonald, and T. K. Alexander, Phys. Rev. C 12, 1158 (1975).

[17] R. G. Markham, S. M. Austin, and M. A. M. Shahabuddin, Nucl. Phys. A 270, 489 (1976).

[18] A. W. Obst and W. J. Braithwaite, Phys. Rev. C 13, 2033 (1976).

[19] D. E. Alburger, Phys. Rev. 118, 235 (1960).

[20] F. Ajzenberg-Selove and P. H. Stelson, Phys. Rev. 120, 500 (1960).

[21] A. W. Obst, T. B. Grandy, and J. L. Weil, Phys. Rev. C 5, 738 (1972).

[22] R. G. H. Robertson, R. A. Warner, and S. M. Austin, Phys. Rev. C 15, 1072 (1977).

[23] D. E. Alburger, Phys. Rev. C 16, 2394 (1977).

[24] M. Chernykh, H. Feldmeier, T. Neff, P. von Neumann-Cosel, and A. Richter, Phys. Rev. Lett. 105, 022501 (2010).
[25] A. E. Stuchbery, EPJ Web Conf. 232, 01001 (2020).

[26] T. Kibédi, G. D. Dracoulis, and A. P. Byrne, Nucl. Instrum. Methods Phys. Res. A 294, 523 (1990).

[27] T. Kibédi, A. E. Stuchbery, G. D. Dracoulis, and K. A. Robertson, EPJ Web Conf. 35, 06001 (2012).

[28] P. Schlüter and G. Soff, At. Data Nucl. Data Tables 24, 509 (1979).

[29] Ch. Hofmann, J. Reinhardt, W. Greiner, P. Schlüter, and G. Soff, Phys. Rev. C 42, 2632 (1990).

[30] J. R. Oppenheimer, Phys. Rev. 60, 150 (1941).

[31] M. E. Rose, Phys. Rev. 76, 678 (1949).

[32] P. Schlüter, G. Soff, and W. Greiner, Phys. Rep. 75, 327 (1981).

[33] M. E. Rose, Phys. Rev. 131, 1260 (1963).

[34] E. K. Warburton, Phys. Rev. 133, B1368 (1964).

[35] K. Halbach and R. F. Holsinger, Part. Accel. 7, 213 (1976).

[36] F. Salvat, J. M. Fernández-Varea, and J. Sempau, PENELOPE2008: A Code System for Monte Carlo Simulation of Electron and Photon Transport, Workshop Proceedings, Barcelona, Spain, 30 June-3 July 2008 (OECD, 2009), https://www.oecdnea.org/science/pubs/2009/nea6416-penelope.pdf.

[37] A. Devlin, Silicon detector characterisation for Beta spectroscopy - from keV conversion electrons to $\mathrm{MeV}$ electron Positron pairs, M.Phil., Deparment of Nuclear Physics, The Australian National University, 2009.

[38] C. N. Davids and T. I. Bonner, Astrophys. J. 166, 405 (1971).

[39] P. Dyer, D. Bodansky, A. G. Seamster, E. B. Norman, and D. R. Maxson, Phys. Rev. C 23, 1865 (1981).

[40] J. F. Ziegler, M. D. Ziegler, and J. P. Biersack, Nucl. Instrum. Methods Phys. Res. B 268, 1818 (2010).

[41] R. Rafiei, R. du Rietz, D. H. Luong, D. J. Hinde, M. Dasgupta, M. Evers, and A. Diaz-Torres, Phys. Rev. C 81, 024601 (2010).

[42] D. H. Luong, M. Dasgupta, D. J. Hinde, R. du Rietz, R. Rafiei, C. J. Lin, M. Evers, and A. Diaz-Torres, Phys. Lett. B 695, 105 (2010).

[43] K. J. Cook, Zeptosecond dynamics of transfer-triggered breakup: mechanisms, timescales, and consequences for fusion, Ph.D. thesis, Dep. of Nucl. Phys., The Australian National University, 2016.

[44] K. J. Cook (unpublished).

[45] T. Kibédi, T. W. Burrows, M. B. Trzhaskovskaya, P. M. Davidson, and C. W. Nestor, Nucl. Instrum. Methods Phys. Res. A 589, 202 (2008).

[46] AveTools, https://www-nds.iaea.org/public/ensdf_pgm/.

[47] M. U. Rajput and T. D. Mac Mahon, Nucl. Instrum. Methods Phys. Res. A 312, 289 (1992).

[48] C. Angulo, M. Arnould, M. Rayet, P. Descouvemont, D. Baye, C. Leclercq-Willain, A. Coc, S. Barhoumi, P. Aguer, C. Rolfs, R. Kunz, J. W. Hammer, A. Mayer, T. Paradellis, S. Kossionides, C. Chronidou, K. Spyrou, S. Degl'Innocenti, G. Fiorentini, B. Ricci, S. Zavatarelli, C. Providencia, H. Wolters, 
J. Soares, C. Grama, J. Rahighi, A. Shotter, and M. LamehiRachti, Nucl. Phys. A 656, 3 (1999).

[49] T. Kibédi, B. M. Alshahrani, A. E. Stuchbery, M. S. Guttormsen, A. Görgen, S. Siem, A. C. Larsen, F. Giacoppo,
E. Sahin, G. M. Tveten, F. L. Bello Garrote, L. C. Campo, T. K. Eriksen, M. Klintefjord, S. Maharramova, H. T. Nyhus, T. G. Tornyi, and T. Renstrøm, The radiative width of the Hoyle state from $\gamma$-ray spectroscopy, Phys. Rev. Lett. (to be published). 